\title{
San Marino
}

National Cancer Institute

\section{Source}

National Cancer Institute. San Marino. NCI Thesaurus. Code C17115.

A country in southern Europe, an enclave in central Italy. 